\title{
An Extension of the Black-Scholes and Margrabe Formulas to a Multiple Risk Economy
}

\author{
Werner Hürlimann \\ FRS Global Switzerland, Zürich, Switzerland \\ E-mail: werner.huerlimann@frsglobal.com \\ Received January 23, 2011; revised February 14, 2011; accepted February 16, 2011
}

\begin{abstract}
We consider an economic model with a deterministic money market account and a finite set of basic economic risks. The real-world prices of the risks are represented by continuous time stochastic processes satisfying a stochastic differential equation of diffusion type. For the simple class of log-normally distributed instantaneous rates of return, we construct an explicit state-price deflator. Since this includes the Black-Scholes and the Vasicek (Ornstein-Uhlenbeck) return models, the considered deflator is called Black-Scholes-Vasicek deflator. Besides a new elementary proof of the Black-Scholes and Margrabe option pricing formulas a validation of these in a multiple risk economy is achieved.
\end{abstract}

Keywords: State-Price Deflator, Option Pricing, Black-Scholes Model, Vasicek Model, Margrabe Formula

\section{Introduction}

The first rigorous mathematical derivation of the BlackScholes formula in [1] (see also [2]) relies on a dynamic delta-hedge portfolio and a risk-free argument of noarbitrage. Later on [3] introduced state-price deflators, which led to the insight that deflator based market valuation using the real-world probability measure is equivalent to market valuation based on a risk-neutral martingale measure.

The present contribution focuses entirely on state-price deflators, which are summarized in Section 2. We consider in Section 3 an economic model that contains a money market account with deterministic continuous-compounded spot rates and a finite set of basic economic risks (interest rates, stocks and equity, property, commodities, inflation, currency, etc.). The real-world prices of these risks are represented by continuous time stochastic processes satisfying a stochastic differential equation of diffusion type. In the simplest situation of log-normally distributed instantaneous rates of return, which includes the Black-Scholes and the Vasicek (Ornstein-Uhlenbeck) return models, we construct in Proposition 3.2 the so-called Black-Scholes-Vasicek (BSV) deflator.

The application of the BSV deflator to option pricing follows in Section 4. Besides a new elementary proof of the (slightly extended) Black-Scholes formula it provides a validation of it in a financial market with multiple eco- nomic risks. The same holds true for Margrabe's formula for a European option to exchange one risky asset for another one.

\section{Valuation with State-Price Deflators}

Let $(\Omega, F, P)$ be a probability space such that $\Omega$ is the sample space, which describes the states of the world, $F$ is the $\sigma$-field of events, and $P$ is the probability measure assigning to any event $E$ in $F$ its probability $P(E)$. At each time $t \geq 0$, the $\sigma$-field $F_{t} \subset F$ denotes the set of events, which describes the information available at time $t$. An adapted process $X$ is a set $\left\{X_{t}\right\}_{t \geq 0}$ such that $X_{t}$ is a random variable with respect to the measurable space $\left(\Omega, F_{t}\right)$.

In continuous time finance one considers adapted price processes $S=\left\{S_{t}\right\}_{t \geq 0}$ such that $S_{t}$ represents the random value at time $t$ of a financial instrument. To place a market value or price on any financial instrument, we consider a (state-price) deflator $D=\left\{D_{t}\right\}_{t \geq 0}$, that is a strictly positive adapted process such that the stochastic value $S_{t}$ payable at time $t$ has value at time $s<t$ given by the formula

$$
S_{s}=D_{s}^{-1} \cdot E_{s}\left[D_{t} S_{t}\right], \quad 0 \leq s<t,
$$

where by convention $E_{s}\left[X_{t}\right]=E_{P}\left[X_{t} \mid F_{s}\right]$ denotes the expected value (under the real-world probability measure 
$P$ ) of $X_{t}$ at time $t$ given the information at time $s$. This means that the adapted deflated or discounted price process $D S=\left\{D_{t} S_{t}\right\}_{t \geq 0}$ is a martingale. Recall that state-price deflators have been introduced in [3], pp. 23 and 97.

\section{The Black-Scholes-Vasicek Deflator for Multiple Economic Risks}

We suppose that the economic model contains

1) A deterministic money market account $M=\left\{M_{t}\right\}_{t \geq 0}$ with value

$$
M_{t}=M_{s} \exp ((t-s) R(s, t)), \quad 0 \leq s<t,
$$

where $R(s, t), 0 \leq s<t$, are the deterministic continuous-compounded spot rates. The price at time $s$ of a zero-coupon bond paying one unit of money at time $t$ (that corresponds to the money market account) is defined and denoted throughout by

$$
P(s, t)=\exp (-(t-s) R(s, t)), \quad 0 \leq s<t .
$$

2) A finite set of $m$ economic risks (interest rates, stocks and equity, property, commodities, inflation, currency, etc.), which are measured by indices. The continuous instantaneous change in each index defines a financial instrument $I_{k}, k=1, \cdots, m$, whose real-world price is represented by a continuous time stochastic process $S^{(k)}=\left\{S_{t}^{(k)}\right\}_{t \geq 0}$, which satisfies a stochastic differential equation

$$
\begin{aligned}
& \mathrm{d} S_{t}^{(k)}=\mu_{k}\left(t, r_{t}^{(k)}\right) S_{t}^{(k)} \mathrm{d} t+\sigma_{k}\left(t, r_{t}^{(k)}\right) S_{t}^{(k)} \mathrm{d} W_{t}^{(k)}, \\
& k=1, \cdots, m,
\end{aligned}
$$

where the instantaneous rate of return $r_{t}^{(k)}$ is assumed to follow a diffusion process

$$
\mathrm{d} r_{t}^{(k)}=\mu_{k}\left(t, r_{t}^{(k)}\right) \mathrm{d} t+\sigma_{k}\left(t, r_{t}^{(k)}\right) \mathrm{d} W_{t}^{(k)}, \quad k=1, \cdots, m,
$$

with $\mu_{k}(t, \cdot)$ the drift, $\sigma_{k}(t, \cdot)$ the instantaneous standard deviation, and $W_{t}^{(k)}$ a standard Wiener process. The standard Wiener processes are correlated such that

$$
E\left[\mathrm{~d} W_{t}^{(i)} \mathrm{d} W_{t}^{(j)}\right]=\rho_{i j} \mathrm{~d} t, \quad 1 \leq i, j \leq m .
$$

The correlation matrix is denoted by $C=\left(\rho_{i j}\right)$ and we assume that it is a valid correlation matrix, i.e. it is positive semi-definite. In general, it is still possible to construct a valid correlation matrix that approximates with respect to a given norm a given invalid correlation matrix (e.g. [4] for the spectral decomposition method). The real-world prices of the financial instruments (1) at a time $t>0$ given the information at a previous time $0 \leq s<t$ can be expressed using an exponential function of a stochastic integral as follows.

Proposition 1 The real-world prices of the financial instruments $I_{k}, k=1, \cdots, m$, satisfy the following stochastic representations

$$
\begin{aligned}
& S_{t}^{(k)}=S_{s}^{(k)} \exp \left\{r_{t}-r_{s}-\frac{1}{2} \int_{s}^{t} \sigma_{k}^{2}\left(u, r_{u}^{(k)}\right) \mathrm{d} u\right\}, \\
& 0 \leq s<t, \quad k=1, \cdots, m .
\end{aligned}
$$

Proof. For fixed $k$ set $X_{t}=\left(r_{t}^{(k)}, S_{t}^{(k)}\right)$ to see that

$$
\mathrm{d} X_{t}=M\left(t, X_{t}\right) \mathrm{d} t+\sum\left(t, X_{t}\right) \mathrm{d} W_{t}^{(k)}
$$

with

$$
\begin{aligned}
& M\left(t, X_{t}\right)=\left(\mu_{k}\left(t, r_{t}^{(k)}\right), S_{t}^{(k)} \mu_{k}\left(t, r_{t}^{(k)}\right)\right), \\
& \sum\left(t, X_{t}\right)=\left(\sigma_{k}\left(t, r_{t}^{(k)}\right), S_{t}^{(k)} \sigma_{k}\left(t, r_{t}^{(k)}\right)\right) .
\end{aligned}
$$

The result follows through application of the bivariate version of Itô's Lemma (e.g. [5], Section 2).

For simplicity, and to describe the main features in an analytical way, we restrict the attention to either BlackScholes return processes $\mathrm{d} r_{t}^{(k)}=\mu_{k} \mathrm{~d} t+\sigma_{k} \mathrm{~d} W_{t}^{(k)}$ (stocks and equity) or Vasicek (Ornstein-Uhlenbeck) return processes $\mathrm{d} r_{t}^{(k)}=a_{k}\left(b_{k}-r_{t}^{(k)}\right) \mathrm{d} t+\sigma_{k} \mathrm{~d} W_{t}^{(k)} \quad$ (interest rates, property, commodities, inflation, currency, etc.). In both cases the return differences $r_{t}^{(k)}-r_{s}^{(k)}, 0 \leq s<t$, are normally distributed, which implies that the prices (6) are lognormally distributed. For a unified analysis let $m_{k}(s, t)$ and $v_{k}(s, t)$ denote the mean and standard deviation per time unit of these return differences as given by

Black-Scholes return model

$$
m_{k}(s, t)=\mu_{k}, \quad v_{k}(s, t)=\sigma_{k}, \quad 0 \leq s<t
$$

Vasicek return model

$$
\begin{aligned}
& m_{k}(s, t)=\frac{\left(b_{k}-r_{s}^{(k)}\right)\left(1-e^{-a_{k}(t-s)}\right)}{t-s}, \\
& v_{k}(s, t)=\sigma_{k} \sqrt{\frac{1-e^{-2 a_{k}(t-s)}}{2 a_{k}(t-s)}}
\end{aligned}
$$

In this situation Proposition 1 yields the following equalities in distribution

$$
S_{t}^{(k)}=S_{s}^{(k)} \exp \left\{\left(m_{k}(s, t)-\frac{1}{2} \sigma_{k}^{2}\right)(t-s)+v_{k} \sqrt{t-s} \cdot W_{t-s}^{(k)}\right\},
$$

$0 \leq s<t, \quad k=1, \cdots, m$,

where the $W_{t-s}^{(k)}$ 's are correlated standard Wiener processes such that $E\left[\mathrm{~d} W_{t-s}^{(i)} \mathrm{d} W_{t-s}^{(j)}\right]=\rho_{i j} \mathrm{~d} t$.

Following Section 2 consider now the Black-ScholesVasicek deflator in the multiple risk economy, for short $B S V$ deflator, which has the same form as the price processes in (9), i.e. 


$$
D_{t}^{(m)}=D_{s}^{(m)} \exp \left\{\alpha^{(m)}(s, t)(t-s)-\beta^{(m)}(s, t)^{T} \sqrt{t-s} \cdot W_{t-s}\right\}, \quad 0 \leq s<t,
$$

for some parametric function $\alpha^{(m)}(s, t)$ and vectors

$$
\begin{gathered}
\boldsymbol{\beta}^{(m)}(s, t)=\left(\beta_{1}^{(m)}(s, t), \cdots, \beta_{m}^{(m)}(s, t)\right)^{T}, \\
\boldsymbol{W}_{t-s}=\left(W_{t-s}^{(1)}, \cdots, W_{t-s}^{(m)}\right)^{T} .
\end{gathered}
$$

To define a state-price deflator the stochastic processes (9) and (10) must satisfy the martingale conditions

$$
\begin{aligned}
& E_{s}\left[D_{t}^{(m)}\right]=D_{s}^{(m)} P(s, t)=D_{s}^{(m)} e^{-(t-s) R(s, t)}, \\
& E_{s}\left[D_{t}^{(m)} S_{t}^{(k)}\right]=D_{s}^{(m)} S_{s}^{(k)}, \quad 0 \leq s<t, \quad k=1, \cdots, m .
\end{aligned}
$$

Proposition 2 (BSV deflator) Given is a financial market with a risk-free money market account and $m$ economic risks that have log-normal real-world prices (9). Assume a non-singular valid correlation matrix $\boldsymbol{C}$ with non-vanishing determinant. Then, the BSV deflator (10) is determined by

$$
\begin{aligned}
D_{t}^{(m)}=D_{s}^{(m)} \exp \{ & -R(s, t)(t-s)-\frac{1}{2} \sum_{j=1}^{m} \beta_{j}^{(m)}(s, t)^{2}(t-s) \\
& -\sum_{1 \leq i<j \leq m} \rho_{i j} \beta_{i}^{(m)}(s, t) \beta_{j}^{(m)}(s, t)(t-s) \\
& \left.-\sum_{j=1}^{m} \beta_{j}^{(m)}(s, t) \sqrt{t-s} \cdot W_{t-s}^{(j)}\right\},
\end{aligned}
$$

$0 \leq s<t$

with

$$
\begin{aligned}
& \beta_{j}^{(m)}(s, t)=\operatorname{det}(\boldsymbol{C})^{-1} \cdot \sum_{i=1}^{m}(-1)^{i+j} \operatorname{det}\left(\boldsymbol{C}_{j}^{(i)}\right) \cdot \lambda_{i}(s, t), \\
& \lambda_{i}(s, t)=\frac{m_{i}(s, t)-R(s, t)-\frac{1}{2}\left(\sigma_{i}^{2}-v_{i}^{2}(s, t)\right)}{v_{i}(s, t)}, \\
& 0 \leq s<t,
\end{aligned}
$$

where $\boldsymbol{C}_{j}^{(i)}$ is the matrix formed by deleting the $i$-th row and $j$-th column of $\boldsymbol{C}$. The quantity $\lambda_{i}(s, t)$ is called market price of the i-th economic risk.

Remark 1 In the Black-Scholes return model the market price of the $i$-th economic risk is given by $\lambda_{i}(s, t)=\left(\mu_{i}-R(s, t)\right) / \sigma_{i} \quad$ (Sharpe ratio).

Proof. The martingale conditions (11) are equivalent with the system of equations

$$
\begin{gathered}
R(s, t)+\alpha^{(m)}(s, t)+\frac{1}{2} \sum_{j=1}^{m} \beta_{j}^{(m)}(s, t)^{2} \\
+\sum_{1 \leq i<j \leq m} \rho_{i j} \beta_{i}^{(m)}(s, t) \beta_{j}^{(m)}(s, t)=0, \quad 0 \leq s<t, \\
\alpha^{(m)}(s, t)+m_{k}(s, t)-\frac{1}{2} \sigma_{k}^{2}+\frac{1}{2}\left(v_{k}(s, t)-\beta_{k}^{(m)}(s, t)\right)^{2} \\
-\left(v_{k}(s, t)-\beta_{k}^{(m)}(s, t)\right) \cdot \sum_{j \neq k} \rho_{j k} \beta_{j}^{(m)}(s, t) \\
+\frac{1}{2} \sum_{j \neq k} \beta_{j}^{(m)}(s, t)^{2}+\sum_{i<j, i, j \neq k} \rho_{i j} \beta_{i}^{(m)}(s, t) \beta_{j}^{(m)}(s, t)=0, \\
0 \leq s<t, \quad k=1, \cdots, m .
\end{gathered}
$$

Insert (14) into (15) using the definition of $\lambda_{i}(s, t)$ to obtain the matrix equation $\boldsymbol{C} \cdot \boldsymbol{\beta}^{(m)}(s, t)=\lambda(s, t)$, where $\lambda(s, t)=\left(\lambda_{1}(s, t), \cdots, \lambda_{m}(s, t)\right)^{T}$ is the market price vector of economic risks. If $\operatorname{Adj}(\boldsymbol{C})$ denotes the adjoint matrix, then by Cramer's rule one has $\boldsymbol{\beta}^{(m)}(s, t)=\operatorname{det}(\boldsymbol{C})^{-1} \cdot \operatorname{Adj}(\boldsymbol{C}) \cdot \lambda(s, t)$, which implies the more explicit formula (13). The expression for the parametric function $\alpha(s, t)$ follows from (14).

Examples 1 For a single economic risk the BSV deflator reads

$$
\begin{aligned}
& D_{t}^{(1)}=D_{s}^{(1)} \exp \left\{-R(s, t)(t-s)-\frac{1}{2} \lambda_{1}^{2}(s, t)(t-s)\right. \\
& \left.-\lambda_{1}(s, t) \sqrt{t-s} \cdot W_{t-s}\right\}, \\
& \lambda_{1}(s, t)=\frac{m_{1}(s, t)-R(s, t)-\frac{1}{2}\left(\sigma_{1}^{2}-v_{1}^{2}(s, t)\right)}{v_{1}(s, t)}, 0 \leq s<t,
\end{aligned}
$$

The other lower dimensional special cases are fully analytical. For $m=2$ one has

$$
\begin{aligned}
& \beta_{1}^{(2)}(s, t)=\frac{\lambda_{1}(s, t)-\rho_{12} \lambda_{2}(s, t)}{1-\rho_{12}^{2}}, \\
& \beta_{2}^{(2)}(s, t)=\frac{\lambda_{2}(s, t)-\rho_{12} \lambda_{1}(s, t)}{1-\rho_{12}^{2}},
\end{aligned}
$$

Similarly, for $m=3$ one has (note for mnemonic purposes the cyclic permutations)

$$
\begin{aligned}
& \beta_{1}^{(3)}(s, t) \cdot \operatorname{det}(\boldsymbol{C})=\lambda_{1}(s, t)\left(1-\rho_{23}^{2}\right)-\lambda_{2}(s, t)\left(\rho_{12}-\rho_{13} \rho_{23}\right)-\lambda_{3}(s, t)\left(\rho_{13}-\rho_{12} \rho_{23}\right), \\
& \beta_{2}^{(3)}(s, t) \cdot \operatorname{det}(\boldsymbol{C})=\lambda_{2}(s, t)\left(1-\rho_{13}^{2}\right)-\lambda_{3}(s, t)\left(\rho_{23}-\rho_{12} \rho_{13}\right)-\lambda_{1}(s, t)\left(\rho_{12}-\rho_{23} \rho_{13}\right), \\
& \beta_{3}^{(3)}(s, t) \cdot \operatorname{det}(\boldsymbol{C})=\lambda_{3}(s, t)\left(1-\rho_{12}^{2}\right)-\lambda_{1}(s, t)\left(\rho_{13}-\rho_{23} \rho_{12}\right)-\lambda_{2}(s, t)\left(\rho_{23}-\rho_{13} \rho_{12}\right), \\
& \operatorname{det}(\boldsymbol{C})=1-\left(\rho_{12}^{2}+\rho_{23}^{2}+\rho_{13}^{2}\right)+2 \rho_{12} \rho_{23} \rho_{13} .
\end{aligned}
$$




\section{The Black-Scholes and Margrabe Formulas in a Multiple Risk Economy}

We begin with an elementary result in probability theory. Suppose that the random vector $\left(S_{1}, S_{2}\right)$ has a bivariate lognormal distribution with parameter vector

$\left(\mu_{1}, v_{1}, \mu_{2}, v_{2}, \rho\right)$ such that the standardized random vector

$$
\left(U_{1}, U_{2}\right)=\left(\frac{\ln S_{1}-\mu_{1}}{v_{1}}, \frac{\ln S_{2}-\mu_{2}}{v_{2}}\right)
$$

has a standard bivariate normal distribution with correlation coefficient $\rho$.

Lemma 1. The expected positive difference of the bivariate lognormal spread is given by

$$
\begin{aligned}
& E\left[\left(S_{1}-S_{2}\right)_{+}\right] \\
& =\exp \left(\mu_{1}+\frac{1}{2} v_{1}^{2}\right) \Phi\left(\frac{\mu_{1}-\mu_{2}+v_{1}^{2}-\rho v_{1} v_{2}}{\sqrt{v_{1}^{2}+v_{2}^{2}-2 \rho v_{1} v_{2}}}\right) \\
& -\exp \left(\mu_{2}+\frac{1}{2} v_{2}^{2}\right) \Phi\left(\frac{\mu_{1}-\mu_{2}-v_{2}^{2}+\rho v_{1} v_{2}}{\sqrt{v_{1}^{2}+v_{2}^{2}-2 \rho v_{1} v_{2}}}\right),
\end{aligned}
$$

with $\Phi(x)$ the standard normal distribution.

Proof. The derivation is left as exercise.

The formula (20) is the unifying mathematical content leading to the (slightly extended) European call option formula by [6] (Theorem 1) (see also [7-9]) and the (slightly extended) formula by [10] for pricing the exchange option (Theorem 2). Both formulas are validated within a multiple risk economy.

Theorem 1 (Black-Scholes in a multiple risk economy) Under the assumptions of Proposition 2, the market value at time $s \geq 0$ of a European call option on the financial instrument $I_{k}, k \in\{1, \cdots, m\}$ with strike time $t>s$ and strike price $K$ is given by the formula

$$
\begin{aligned}
E_{s} & {\left[D_{t}^{(m)}\left(S_{t}^{(k)}-K\right)_{+}\right] } \\
= & D_{s}^{(m)} \cdot\left(S_{s}^{(k)} \cdot \Phi\left(d_{1}^{(k, m)}(s, t)\right)\right. \\
& \left.-K \cdot P(s, t) \cdot \Phi\left(d_{2}^{(k, m)}(s, t)\right)\right)
\end{aligned}
$$

with

$$
\begin{aligned}
& d_{1}^{(k, m)}(s, t)=\frac{\ln \left(S_{s}^{(k)} / K\right)+\left(R(s, t)+\frac{1}{2} v_{k}^{2}(s, t)\right)(t-s)}{v_{k}(s, t) \sqrt{t-s}}, \\
& d_{2}^{(k, m)}(s, t)=d_{1}^{(k, m)}(s, t)-v_{k}(s, t) \sqrt{t-s}, \\
& 0 \leq s<t, \quad k=1, \cdots, m .
\end{aligned}
$$

Remark 2 If for $m=1$ one specializes to the BlackScholes single risk economy with constant risk-free return $R(s, t)=r>0$ and Black-Scholes return model with constant volatility $v_{1}(s, t)=\sigma_{1}$, one recovers the original formula in [6].

Proof. This is an application of Lemma 1. We distinguish between two cases. If $m=1$ one writes using (14) that $D_{t}^{(1)}\left(S_{t}^{(1)}-K\right)_{+}=\left(e^{\mu_{1}+v_{1} U_{1}}-e^{\mu_{2}+v_{2} U_{2}}\right)_{+}$with

$$
\begin{gathered}
\mu_{1}=\ln D_{s}^{(1)}-R(s, t)(t-s)-\frac{1}{2} \lambda_{1}^{2}(s, t)(t-s) \\
+\ln S_{s}^{(1)}+\left(m_{1}(s, t)-\frac{1}{2} \sigma_{1}^{2}\right)(t-s), \\
\mu_{2}=\ln D_{s}^{(1)}-R(s, t)(t-s)-\frac{1}{2} \lambda_{1}^{2}(s, t)(t-s)+\ln K, \\
v_{1}=\left|v_{1}(s, t)-\lambda_{1}(s, t)\right| \sqrt{t-s}, \quad v_{2}=\lambda_{1}(s, t) \sqrt{t-s}, \\
U_{1}=\operatorname{sgn}\left(v_{1}(s, t)-\lambda_{1}(s, t)\right) \cdot W_{t-s}^{(1)}, \quad U_{2}=-W_{t-s}^{(1)}, \\
\rho=-\operatorname{sgn}\left(v_{1}(s, t)-\lambda_{1}(s, t)\right) .
\end{gathered}
$$

Through elementary algebra one sees that (use the definition of $\lambda_{1}(s, t)$ in (13))

$$
\begin{aligned}
& v_{1}^{2}+v_{2}^{2}-2 \rho v_{1} v_{2}=v_{1}^{2}(s, t)(t-s), \\
& \mu_{1}-\mu_{2}+v_{1}^{2}-\rho v_{1} v_{2} \\
& =\ln \left(S_{s}^{(1)} / K\right)+\left(R(s, t)+\frac{1}{2} v_{1}^{2}(s, t)\right)(t-s), \\
& \mu_{1}-\mu_{2}-v_{2}^{2}+\rho v_{1} v_{2} \\
& \quad=\ln \left(S_{s}^{(1)} / K\right)+\left(R(s, t)-\frac{1}{2} v_{1}^{2}(s, t)\right)(t-s), \\
& \mu_{1}+\frac{1}{2} v_{1}^{2}=\ln D_{s}^{(1)}+\ln S_{s}^{(1)}, \\
& \mu_{2}+\frac{1}{2} v_{2}^{2}=\ln D_{s}^{(1)}+\ln K-R(s, t)(t-s) .
\end{aligned}
$$

Plugging into (20) one obtains (21). If $m \geq 2$ it suffices (for reasons of symmetry) to show (22) for $k=1$. For this consider the quantities $\bar{\beta}_{2}^{(m)}(s, t), \bar{\rho}^{(m)}$ defined by

$$
\begin{aligned}
& \bar{\beta}_{2}^{(m)}(s, t)^{2}=\sum_{j=2}^{m} \beta_{j}^{(m)}(s, t)^{2} \\
& +\sum_{2 \leq i<j \leq m} \rho_{i j} \beta_{i}^{(m)}(s, t) \beta_{j}^{(m)}(s, t), \\
& \bar{\rho}^{(m)} \bar{\beta}_{2}^{(m)}(s, t)=\sum_{j=2}^{m} \rho_{1 j} \beta_{j}^{(m)}(s, t) .
\end{aligned}
$$

From the proof of Proposition 2 one has $\lambda(s, t)=\boldsymbol{C} \cdot \boldsymbol{\beta}^{(m)}(s, t)$, which in particular implies the identity $\lambda_{1}(s, t)=\beta_{1}^{(m)}(s, t)+\bar{\rho}^{(m)} \bar{\beta}_{2}^{(m)}(s, t)$, which is used several times below. Using (12) one has $D_{t}^{(m)}\left(S_{t}^{(1)}-K\right)_{+}=\left(e^{\mu_{1}+v_{1} U_{1}}-e^{\mu_{2}+v_{2} U_{2}}\right)_{+}$with 


$$
\begin{gathered}
\mu_{1}=\ln D_{s}^{(m)}-\left(R(s, t)+\frac{1}{2} A(s, t)\right)(t-s)+\ln S_{s}^{(1)}+\left(m_{1}(s, t)-\frac{1}{2} \sigma_{1}^{2}\right)(t-s), \\
\mu_{2}=\ln D_{s}^{(m)}-\left(R(s, t)+\frac{1}{2} A(s, t)\right)(t-s)+\ln K, \\
A(s, t)=\beta_{1}^{(m)}(s, t)^{2}+\bar{\beta}_{2}^{(m)}(s, t)^{2}+2 \bar{\rho}^{(m)} \beta_{1}^{(m)}(s, t) \bar{\beta}_{2}^{(m)}(s, t), \\
v_{1}^{2}=\left(A(s, t)+v_{1}(s, t)^{2}-2 v_{1}(s, t) \lambda_{1}(s, t)\right) \cdot(t-s), \quad v_{2}^{2}=A(s, t) \cdot(t-s), \\
v_{1} U_{1}=\left(v_{1}(s, t)-\beta_{1}^{(m)}(s, t)\right) \sqrt{t-s} \cdot W_{t-s}^{(1)}-\sum_{j=2}^{m} \beta_{j}^{(m)}(s, t) \sqrt{t-s} W_{t-s}^{(j)}, \\
v_{2} U_{2}=-\beta_{1}^{(m)}(s, t) \sqrt{t-s} \cdot W_{t-s}^{(1)}-\sum_{j=2}^{m} \beta_{j}^{(m)}(s, t) \sqrt{t-s} W_{t-s}^{(j)}, \\
\rho v_{1} v_{2}=\operatorname{Cov}\left[v_{1} U_{1}, v_{2} U_{2}\right]=\left(A(s, t)-v_{1}(s, t) \lambda_{1}(s, t)\right) \cdot(t-s) .
\end{gathered}
$$

Elementary algebra shows the relations (use the definition of $\lambda_{1}(s, t)$ in (13))

$$
\begin{aligned}
& v_{1}^{2}+v_{2}^{2}-2 \rho v_{1} v_{2}=v_{1}^{2}(s, t)(t-s), \\
& \mu_{1}-\mu_{2}+v_{1}^{2}-\rho v_{1} v_{2} \\
&= \ln \left(S_{s}^{(1)} / K\right)+\left(R(s, t)+\frac{1}{2} v_{1}^{2}(s, t)\right)(t-s), \\
& \mu_{1}-\mu_{2}-v_{2}^{2}+\rho v_{1} v_{2} \\
&=\ln \left(S_{s}^{(1)} / K\right)+\left(R(s, t)-\frac{1}{2} v_{1}^{2}(s, t)\right)(t-s), \\
& \mu_{1}+\frac{1}{2} v_{1}^{2}=\ln D_{s}^{(m)}+\ln S_{s}^{(1)}, \\
& \mu_{2}+\frac{1}{2} v_{2}^{2}=\ln D_{s}^{(m)}+\ln K-R(s, t)(t-s) .
\end{aligned}
$$

Inserting into (20) one obtains (21) for $k=1$.

Theorem 2 (Margrabe in a multiple risk economy) Under the assumptions of Proposition 2, the market value at time $s \geq 0$ of a European exchange option on the financial instruments $I_{k}, I_{\ell}, k \neq \ell \in\{1, \cdots, m\}$ with strike time $t>s$ is given by the formula

$$
\begin{aligned}
& E_{s}\left[D_{t}^{(m)}\left(S_{t}^{(k)}-S_{t}^{(\ell)}\right)_{+}\right]= \\
& D_{s}^{(m)}\left(S_{s}^{(k)} \Phi\left(\frac{\ln \left(S_{s}^{(k)} / S_{s}^{(\ell)}\right)+\frac{1}{2} v^{2}(s, t)(t-s)}{v(s, t) \sqrt{t-s}}\right)\right. \\
& \left.\quad-S_{s}^{(\ell)} \Phi\left(\frac{\ln \left(S_{s}^{(k)} / S_{s}^{(\ell)}\right)-\frac{1}{2} v^{2}(s, t)(t-s)}{v(s, t) \sqrt{t-s}}\right)\right), \\
& v^{2}(s, t)=v_{k}^{2}(s, t)+v_{\ell}^{2}(s, t)-2 \rho_{k \ell} v_{k}(s, t) v_{\ell}(s, t), \\
& 0 \leq s<t, \quad k \neq \ell \in\{1, \cdots, m\} .
\end{aligned}
$$

Proof. For reasons of symmetry it suffices to show (23) for $k=1, \ell=2$. Consider the quantities $\bar{\beta}_{3}^{(m)}(s, t)$, $\bar{\rho}_{1}^{(m)}, \bar{\rho}_{2}^{(m)}$ defined by (if $m=2$ the sums are empty and the quantities zero)

$$
\begin{aligned}
& \bar{\beta}_{3}^{(m)}(s, t)^{2}=\sum_{j=3}^{m} \beta_{j}^{(m)}(s, t)^{2} \\
& +\sum_{3 \leq i<j \leq m} \rho_{i j} \beta_{i}^{(m)}(s, t) \beta_{j}^{(m)}(s, t), \\
& \bar{\rho}_{1}^{(m)} \bar{\beta}_{3}^{(m)}(s, t)=\sum_{j=3}^{m} \rho_{1 j} \beta_{j}^{(m)}(s, t), \\
& \bar{\rho}_{2}^{(m)} \bar{\beta}_{3}^{(m)}(s, t)=\sum_{j=3}^{m} \rho_{2 j} \beta_{j}^{(m)}(s, t) .
\end{aligned}
$$

Since $\lambda(s, t)=C \cdot \beta^{(m)}(s, t)$ (proof of Proposition 2) one has in particular the identities

$$
\begin{aligned}
& \lambda_{1}(s, t)=\beta_{1}^{(m)}(s, t)+\rho_{12} \beta_{2}^{(m)}(s, t)+\bar{\rho}_{1}^{(m)} \bar{\beta}_{3}^{(m)}(s, t), \\
& \lambda_{2}(s, t)=\rho_{12} \beta_{1}^{(m)}(s, t)+\beta_{2}^{(m)}(s, t)+\bar{\rho}_{2}^{(m)} \bar{\beta}_{3}^{(m)}(s, t) .
\end{aligned}
$$

Using (12) one writes

$$
D_{t}^{(m)}\left(S_{t}^{(1)}-S_{t}^{(2)}\right)_{+}=\left(e^{\mu_{1}+v_{1} U_{1}}-e^{\mu_{2}+v_{2} U_{2}}\right)_{+}
$$

with

$$
\begin{aligned}
\mu_{i}=\ln & D_{s}^{(m)}-\left(R(s, t)+\frac{1}{2} A(s, t)\right)(t-s) \\
+ & \ln S_{s}^{(i)}+\left(m_{i}(s, t)-\frac{1}{2} \sigma_{i}^{2}\right)(t-s), \quad i=1,2, \\
A(s, t)= & \beta_{1}^{(m)}(s, t)^{2}+\beta_{2}^{(m)}(s, t)^{2}+\bar{\beta}_{3}^{(m)}(s, t)^{2} \\
& +2 \rho_{12} \beta_{1}^{(m)}(s, t) \beta_{2}^{(m)}(s, t) \\
& +2 \bar{\rho}_{1}^{(m)} \beta_{1}^{(m)}(s, t) \bar{\beta}_{3}^{(m)}(s, t) \\
& +2 \bar{\rho}_{2}^{(m)} \beta_{2}^{(m)}(s, t) \bar{\beta}_{3}^{(m)}(s, t)
\end{aligned}
$$




$$
\begin{aligned}
& v_{1}^{2}=\left(A(s, t)+v_{1}(s, t)^{2}-2 v_{1}(s, t) \lambda_{1}(s, t)\right) \cdot(t-s), \\
& v_{2}^{2}=\left(A(s, t)+v_{2}(s, t)^{2}-2 v_{2}(s, t) \lambda_{2}(s, t)\right) \cdot(t-s) \text {, } \\
& v_{1} U_{1}=\left(v_{1}(s, t)-\beta_{1}^{(m)}(s, t)\right) \sqrt{t-s} \cdot W_{t-s}^{(1)} \\
& -\beta_{2}^{(m)}(s, t) \sqrt{t-s} \cdot W_{t-s}^{(2)}-\sum_{j=3}^{m} \beta_{j}^{(m)}(s, t) \sqrt{t-s} W_{t-s}^{(j)}, \\
& v_{2} U_{2}=-\beta_{1}^{(m)}(s, t) \sqrt{t-s} \cdot W_{t-s}^{(1)} \\
& +\left(v_{2}(s, t)-\beta_{2}^{(m)}(s, t)\right) \sqrt{t-s} \cdot W_{t-s}^{(2)} \\
& -\sum_{j=3}^{m} \beta_{j}^{(m)}(s, t) \sqrt{t-s} W_{t-s}^{(j)}, \\
& \rho v_{1} v_{2}=\operatorname{Cov}\left[v_{1} U_{1}, v_{2} U_{2}\right] \\
& =\left(A(s, t)-v_{1}(s, t) \lambda_{1}(s, t)-v_{2}(s, t) \lambda_{2}(s, t)\right. \\
& \left.+\rho_{12} v_{1}(s, t) v_{2}(s, t)\right) \cdot(t-s) \text {. }
\end{aligned}
$$

One obtains the relations (use again the definition of $\lambda_{i}(s, t), i=1,2$ in (13))

$$
\begin{aligned}
v_{1}^{2} & +v_{2}^{2}-2 \rho v_{1} v_{2} \\
& =\left(v_{1}^{2}(s, t)+v_{2}^{2}(s, t)-2 \rho_{12} v_{1}(s, t) v_{2}(s, t)\right) \cdot(t-s) \\
& =v^{2}(s, t) \cdot(t-s) \\
\mu_{1} & -\mu_{2}+v_{1}^{2}-\rho v_{1} v_{2} \\
& =\ln \left(S_{s}^{(1)} / S_{s}^{(2)}\right)+\frac{1}{2} v^{2}(s, t) \cdot(t-s), \\
\mu_{1} & -\mu_{2}-v_{2}^{2}+\rho v_{1} v_{2} \\
& =\ln \left(S_{s}^{(1)} / S_{s}^{(2)}\right)-\frac{1}{2} v^{2}(s, t) \cdot(t-s), \\
\mu_{i} & +\frac{1}{2} v_{i}^{2}=\ln D_{s}^{(m)}+\ln S_{s}^{(i)}, \quad i=1,2 .
\end{aligned}
$$

Inserting into (20) one obtains the Formula (23) for $k=1, \ell=2$.

It might be useful to conclude with a short summary. If one starts with the stochastic representation (9) of the real-world prices for the risks in the economy, the derivation of the formulas is rather elementary. It only uses introductory Probability Theory (including the notion of
Martingale) and Linear Algebra. Therefore, the proof is accessible to any knowledgeable person in these mathematical areas. Moreover, the approach is different from the original one (hedging argument, use of Itô's Lemma and solution of a partial differential equation). It leads to new insight in Option Pricing Theory. Besides a general validation in a multiple risk economy, the proposed derivation implies a risk-neutral property of independent interest, i.e. the formulas are invariant with respect to the market prices of the risk factors.

\section{References}

[1] R. C. Merton, “Theory of Rational Option Pricing,” Bell Journal of Economics and Management Science, Vol. 4, No. 8, 1973, pp. 141-183. Reprinted in [2]. doi: $10.2307 / 3003143$

[2] R. C. Merton, “Continuous-Time Finance,” Basil Blackwell, 1990.

[3] D. Duffie, “Dynamic Asset Pricing Theory,” Princeton University Press, New Jersey, 1992.

[4] R. Rebonato and P. Jäckel, "The Most General Methodology to Create a Valid Correlation Matrix for Risk Management and Option Pricing Purposes,” Journal of Risk, Vol. 2, No. 2, 2000, pp. 17-27.

[5] W. Hürlimann, "Méthodes Stochastiques D'évaluation du Rendement," Proceedings of the $3^{\text {rd }}$ International AFIR Colloquium, Rom, Vol. 2, 1993, pp. 629-649.

[6] F. Black and M. Scholes, "The Pricing of Options and Corporate Liabilities,” Journal of Political Economy, Vol. 81, No. 3, 1973, pp. 637-59. Reprinted in [7-9]. doi:10.1086/260062

[7] M. C. Jensen (Editor), "Studies in the Theory of Capital Market,” Praeger, New York, 1972.

[8] D. L. Luskin (Editor), "Portfolio Insurance: A Guide to Dynamic Hedging,” John Wiley, New York, 1988.

[9] L. Hugston (Editor), "Options: Classic Approaches to Pricing and Modelling,” Risk Books, London, 1999.

[10] W. Margrabe, "The Value of an Option to Exchange one Asset for Another,” Journal of Finance, Vol. 33, No. 1, pp. 177-186, 1978. Reprinted in [9]. doi: $10.2307 / 2326358$ 